\title{
Crystal size and crystalline volume fraction effects on the Erbium emission of nc- Si:Er grown by r.f. sputtering
}

\author{
M. F. Cerqueira*, M. Stepikhova ${ }^{2}$, A. Kozanecki ${ }^{3}$ G. Andrês ${ }^{1}$, E. $^{1}$ Alves $^{4}$ \\ ${ }^{1}$ Departamento de Física, Universidade do Minho, Campus de Gualtar 4710-057 Braga, Portugal \\ ${ }^{2}$ Institute for Physics of Microstructures RAS, 603600 Nizhnij Novgorod GSP-105, Russia \\ ${ }^{3}$ Polish Academy of Sciences, Institute of Physics, PL-02668, Warsaw, Poland \\ ${ }^{4}$ Instituto Técnico Nuclear ITN, EN 10, 2686-953, Sacavém, Portugal
}

*E-mail: fcerqueira@ fisica.uminho.pt, Telf: +253604332; Fax: +253678981

Erbium-doped low-dimensional Si films with different microstructures were grown by reactive magnetron sputtering on glass substrates by varying the deposition parameters. Their structure and chemical composition were studied by micro-Raman and Rutherford backscattering spectrometry, respectively. In this contribution the Erbium emission is studied as a function of nanocrystalline fraction and average crystal sizes and also as a function of the matrix chemical composition. We discuss the temperature dependence of the $\mathrm{Er}^{3+}$ emission as well as the possible explanations of the low Er active fraction.

\section{Introduction}

Intrinsic nanocrystalline silicon (nc-Si) and Er- doped nanocrystalline silicon have attracted enormous interest of researchers as promising candidates for the realization of Si-based visible and infrared light emitters [1]. Er-doped silicon takes special significance for optical communication systems due to the emission at $1.54 \mu \mathrm{m}$. Therefore erbium-doped silicon is the most desired material, for its compatibility with standard CMOS technology, for light sources operating at telecommunications wavelengths. However, the emission efficiency from bulk crystalline c-Si:Er light emitting diodes operating under forward bias is still low, because the major problem - the strong temperature quenching of the Er 
emission [2] has not been solved yet. The situation may be considerably improved by incorporation of Er ions into nanocrystalline silicon. This idea is based on the band-gap widening of a nanometer size $\mathrm{Si}$ crystallites, which consequently will result in reduction of thermal quenching of Er luminescence.

Extensive research work has been devoted in last years to nc-Si thin films intentionally doped with Er. Evidence has been reported that Er-doped nc-Si thin films reveal enhanced intra-ionic luminescence and reduced thermal quenching with respect to crystalline Si [3-6]. The reported data point to excitons confined in nc-Si acting as efficient sensitizers for the rare earth luminescence. However, some controversial results have been obtained, namely concerning the Er radiative lifetime and quenching mechanisms [7], and the fact that only a small fraction of the erbium present in samples is optically active [8-10]. Furthermore D. Kuritsyn et al paid attention to some limitations which are inherent for the system containg Si-clusters embedded in silica matrix and Er ions, such as the lack of resonance of the Er levels and excitons in nc-Si, and spatial distribution of nanocrystals and Er ions limiting energy transfer to Er ions.

In this study the effect of microstructure (average crystal size and crystalline volume fraction) and matrix chemical composition (film hydrogen and oxygen content) on the Erbium emission in as-grown samples produced by sputtering is investigated. Using the sputtering method we produce large area samples at reasonable deposition rates avoiding handling of hazardous gases or chemicals and the hydrogen content can be easily controlled.

\section{Experimental}

Erbium doped silicon thin films were deposited by r.f. reactive magnetron sputtering in an $\mathrm{Ar} / \mathrm{H}_{2}$ atmosphere on ordinary glass substrates under several different conditions using a procedure similar to that applied for the preparation of undoped nc-Si:H films [11], but adding small pieces of metallic erbium to the c-Si target, outside the erosion area. Nanocrystalline samples were grown in a $\mathrm{H}_{2}$ rich 
atmosphere, where the role of atomic hydrogen is to etch preferentially the amorphous phase and promote the amorphous-to-crystalline transition [12].

We have produced silicon thin films having silicon nanocrystals with crystal sizes lower than $5 \mathrm{~nm}$ and around $7 \mathrm{~nm}$ by varying the experimental parameters. The chemical composition was determined by combining Rutherford Backscattering Spectrometry (RBS) and Elastic Recoil Detection (ERD) techniques. For the structural characterisation, Raman scattering measurements were performed in a Jobin Ivon MicroRaman spectrometer with a triple monochromator using the $514.5 \mathrm{~nm}$ line of an argon laser. A liquid nitrogen-cooled CCD was used to detect the scattered light.

Photoluminescence (PL) measurements in the infrared spectral region were performed with a SPEX75 monochromator. The signal was detected using a liquid nitrogen cooled germanium detector, and the $514.5 \mathrm{~nm}$ line of an $\mathrm{Ar}^{+}$laser (laser power on the sample was $150 \mathrm{~mW}$ ) was employed for the excitation. The Er-related PL was studied in the temperature range of $6-300 \mathrm{~K}$.

Since the amount of oxygen and hydrogen in the samples is different for different growth conditions, the chemical composition of the matrix is also different, ranging from purely hydrogenated amorphous silicon $(\mathrm{a}-\mathrm{Si}: \mathrm{H})$ to oxygen rich silicon $(\mathrm{a}-\mathrm{Si}: \mathrm{H}: \mathrm{O})$. In this work we investigate the influence of the matrix chemical composition on the $\mathrm{Er}^{3+} \mathrm{PL}$ efficiency. We also intend to clarify the role of crystal size and crystalline volume fraction on the $\mathrm{Er}^{3+} \mathrm{PL}$ efficiency.

\section{Results and discussion}

\subsection{Structural and chemical characterisation}

Figure 1 shows the Raman spectra of the nc-Si:H samples. The broad band (at around $480 \mathrm{~cm}^{-1}$ ) is assigned to the silicon amorphous matrix and is present in all samples. The presence of Si nanocrystals is revealed by another contribution on the Raman spectra in the vicinity of $521 \mathrm{~cm}^{-1}$. This peak is 
attributed to the transverse optical mode (TO) of crystalline silicon and is shifted to smaller wave numbers for decreasing nanocrystal diameter due to spatial confinement effects [13].

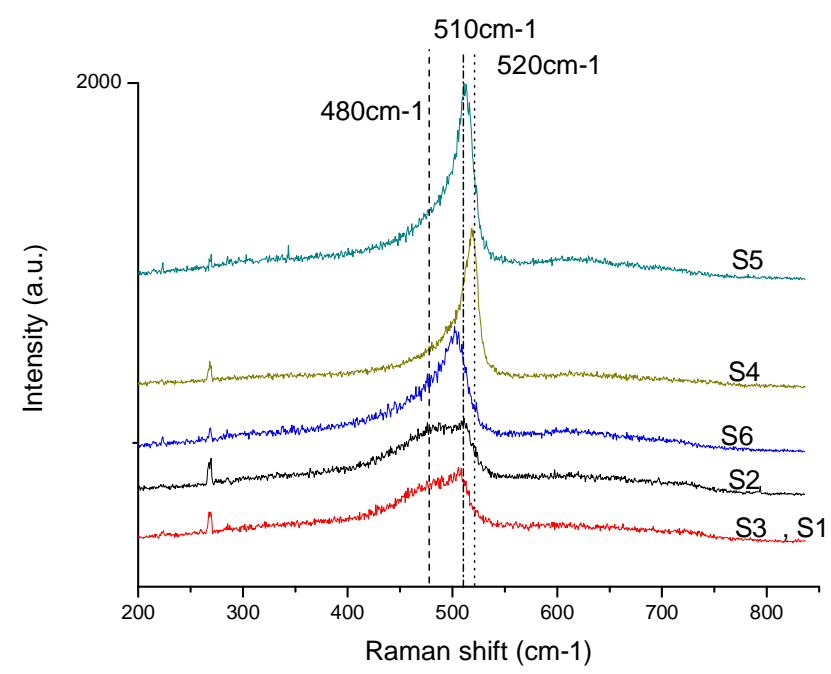

Figure 1 - Raman spectra for samples with different crystallinity.

The samples produced have different microstructures, as revealed by the different intensity ratios of the amorphous and crystalline phases in the Raman spectra (see Fig. 1), and also by the different peak position of the crystalline component $[13,14]$. It is seen that S1, S2and S3 samples show a small crystalline contribution and a stronger amorphous contribution when compared with the other samples. Otherwise the crystalline peak is well defined for the S4, S5 and S6 samples although its position varies between samples.

To analyze the Raman spectra, a computer simulation was performed, assuming that the spectral profile is a superposition of the spectral profiles originating from amorphous and crystalline phases. The crystalline profile was calculated based on the Strong Phonon Confinement [13] model and a Gaussian profile was attributed to the amorphous TO structure. To determine the crystalline volume fraction, $\mathrm{C}$, we use: 


$$
C=\frac{I_{c}}{I_{c}+I_{a}\left(0.1+e^{-D / 25}\right)}
$$

where $I_{c}$ is the integrated intensity of the crystalline component, $I_{a}$ is the integrated intensity of the amorphous peak and 25 is a constant expressed in nm [11]. The average size of the crystallites, $D$, has also to be taken into account since it can influence the value of the scattering cross section. The structural and compositional results of the samples are presented in Table I.

Table I: Properties of samples prepared by reactive magnetron sputtering. D and C are the average crystal size and crystalline volume fraction, respectively, obtained by Raman.

\begin{tabular}{|c|c|c|c|c|c|c|}
\hline Sample & $\begin{array}{c}\mathbf{E r} \\
(\mathbf{a t} \%)\end{array}$ & $\begin{array}{c}\mathbf{H} \\
(\mathbf{a t} \%)\end{array}$ & $\begin{array}{c}\mathbf{O} \\
(\mathbf{a t})\end{array}$ & $\begin{array}{c}\text { Si } \\
(\mathbf{a t} \%)\end{array}$ & $\begin{array}{c}\mathbf{D} \\
(\mathbf{n m})\end{array}$ & $\begin{array}{c}\mathbf{C} \\
(\mathbf{\%})\end{array}$ \\
\hline S1 & 0.03 & 27.5 & $<4$ & 72 & $<3$ & - \\
\hline S2 & 0.55 & 19.1 & 9 & 70 & 4 & - \\
\hline S3 & 0.55 & 19.9 & $<4$ & 70 & 4 & - \\
\hline S6 & 1.81 & 11.5 & 15 & 71 & 7.1 & 31 \\
\hline S5 & 0.89 & 8.9 & 16 & 74 & 7.5 & 61 \\
\hline S4 & 0.6 & 20.9 & 10 & 65 & 7.9 & 62 \\
\hline
\end{tabular}

As can be noticed from Table I, the samples have different $\mathrm{H} / \mathrm{O}$ concentration ratios and also different microstructures reflected by the Raman spectra, namely we produced samples with very small Si NCs sizes having residual oxygen content (S1, S3), and with oxygen content-not residual- (S2) as also samples with high Si NCs sizes having large oxygen content (S4, S5 and S6).

In a previous work [15], concerning the study of the film (matrix) chemical composition, based on the different pseudo dielectric response of the samples, we observed that the difference in the film chemical composition, i.e. different oxygen and hydrogen content, directly results in different matrix 
chemical composition (hydrogenated amorphous silicon, a-Si:H, and oxygen rich hydrogenated amorphous silicon, a-Si:H:O) where the nanocrystals are embedded. We reported that: i) for thin films with residual oxygen content, the silicon crystallites are embedded in a-Si:H matrix; ii) for samples with large $\mathrm{O}$ content, the silicon crystallites are embedded in an oxygen rich amorphous matrix We must then group our samples not only by the crystal sizes: small crystal sizes $(<5 \mathrm{~nm}$ : S1, S2 and S3 samples) and larger crystal sizes (>7nm: S4, S5 and S6 samples), but also by the matrix kind: aSi:H (S1 and S3 samples) and oxygen rich a-Si:H (a-Si:H:O; S2, S4, S5 and S6 samples).

\subsection{Photoluminescence study}

Figure 2 show the low temperature near infrared PL spectra of the erbium doped silicon films with different crystallinity and crystallite sizes, as determined by Raman data (reported in Table I). All the samples studied reveal the PL peak assigned to the ${ }^{4} \mathrm{I}_{13 / 2} \rightarrow{ }^{4} \mathrm{I}_{15 / 2}$ intra-4f transition of $\mathrm{Er}^{3+}$ ions. Furthermore the $\mathrm{Er}^{3+}$ emission is higher for the samples having Si crystal sizes lower than $5 \mathrm{~nm}$ and low crystalline volume fraction (S1, S2 and S3 samples) comparatively to the samples with larger crystal sizes (> 7nm) and higher crystalline volume fraction (S5, S6 and S4 samples- see Fig. 2-inset). Moreover the highest Er PL intensity was obtained for the sample with the lowest Er content (S1 sample). Apart from the low $\mathrm{Er}^{3+}$ emission intensity (efficiency) from samples with crystal sizes larger than 7nm (S5, S6 and S4 samples), it can also be noticed that the PL intensity strongly decreases with increasing crystalline volume fraction.

$\mathrm{The}^{3+} \mathrm{PL}$ spectra are essentially independent of the matrix chemical composition. Comparing samples S2 and S4, that beside the same Er content have the same oxygen and hydrogen amount, i.e. the same matrix kind (a-Si:H:O), S4 sample almost does not show $\mathrm{Er}^{3+}$ emission, then we can conclude that the matrix kind is not the main factor on the Er emission efficiency. Since the difference between 
these two samples is the microstructure and S2 sample presents smaller sizes/lower crystalline volume fraction than S4 sample, we infer that the energy transfer from Si nanocrystals to the Er ions is more efficient for samples having smaller sizes/lower crystalline volume fraction.

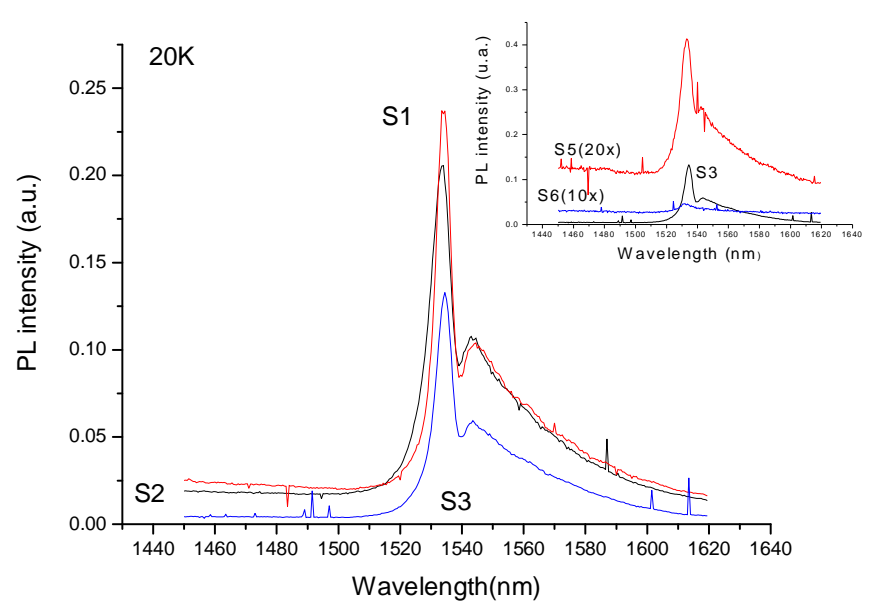

Figure 2 - PL spectra at $\sim 1.54 \mu \mathrm{m}$ measured at $20 \mathrm{~K}$. Inset: $\mathrm{Er}^{3+}$ photoluminescence for the more crystalline samples and S3 sample for comparison.

Another factor that is important is the Er amount on the samples. The first conclusion, based on our results, is that the highest $\mathrm{Er}^{3+} \mathrm{PL}$ intensity was obtained in the sample with lowest erbium amount (S1). We are able to conclude then that the Er concentration needed for obtaining the $\mathrm{Er}^{3+}$ emission is equal or lower than 0.03 at\%. Our results also show that there is no direct correlation between $\mathrm{Er}^{3+}$ PL intensity and the Er content in the sample (see in Fig 2 the spectra of S3, S5 and S6 samples having different Er amount and samples S2, S3 and S4 having the same Er amount).

In order to clarify the correlation between the Er amount and the $\mathrm{Er}^{3+} \mathrm{PL}$, we will focus now our discussion on the PL intensity for samples with Er content around 0.06at\%, namely S2, S3 and S4. It is visible that the $\mathrm{Er}^{3+} \mathrm{PL}$ intensity is not related to the Er content, as we have already mentioned, since the PL intensity decreases from S2 to S3 and strongly to S4 sample and, in all the cases, the $\mathrm{Er}^{3+} \mathrm{PL}$ intensity is lower than for sample S1 which have 0.03 at $\%$ of Er. If we accept the model proposed by 
P.G. Kik [16], that one Si nanocrystal excites one or two Er ions, no reason for this behaviour is found, since the Er amount is the same in all the samples and the PL intensity is high for S2 sample which have the lower crystalline volume fraction.

Otherwise, since the erbium photoluminescence intensity $\left(I_{P L}\right)$ is proportional to the concentration of optically active Er ions $\left(\mathrm{N}_{\mathrm{Er}}\right)$, multiplied by the emission rate $\left(1 / \tau_{\mathrm{Er}}\right)$, the decrease of the erbium emission, can be due to the decrease in the density of radiative centers and/or to the change of coupling between the Er ions and the nc-Si. According to our results on these samples, which have the same Er content, the neighbouring distance between Er ions and silicon NCs does not appear to be the explanation for the low intensity luminescence (small fraction of Er optically active ions), since the number of Si NCs is larger on sample S4, compared with S2 and S3.

Could the transfer of excitation between two neighbouring ions (excitation migration and consequent non-radiative de-excitation) be responsible for the low $\mathrm{Er}^{3+}$ emission when the $\mathrm{Er}$ amount is higher than 0.03 at $\%$ ? The nearest neighbour distance is correlated with the concentration by the expression $R_{n n}=0.55 n^{-1 / 3}$ and, according to Foerster theory, the probability of energy transfer from one excited erbium ion to a ground state one is strongly dependent on $R^{-6}$ ( $R$ is the mutual distance), then, since the Er amount is the same on these samples (S2, S3 and S4), the decrease of intensity cannot be assigned to de-excitation mechanisms (traps, up-conversion or back transfer to the nanocrystal) related with excitation migration[17].

Furthermore, for these samples it is seen that the $\mathrm{Er}^{3+} \mathrm{PL}$ decreases when the crystalline volume fraction/crystal size increases. This behaviour must then be related to the increase of non-radiative channels in sample S4, or to the formation of Si NCs agglomerations/clusters, when the crystalline volume fraction is high, confirming that the spatial distribution of nanocrystals could limit the energy 
transfer to Er ions [8]. The presence of non-radiative path ways is probably related with the existence of more grain boundaries (GB) that could absorb/trap the excitation.

More insight into the PL features was achieved by analyzing the temperature effect on the Er emission. Figure 3a shows room temperature PL of the same samples and Figure $3 b$ shows the corresponding temperature behavior of the $\mathrm{Er}^{3+}$ photoluminescence. The intensity of the erbium emission strongly decreases with increasing temperature (for temperatures higher than $250 \mathrm{~K}$ ), giving information that thermal quenching mechanisms of the Er PL are active.

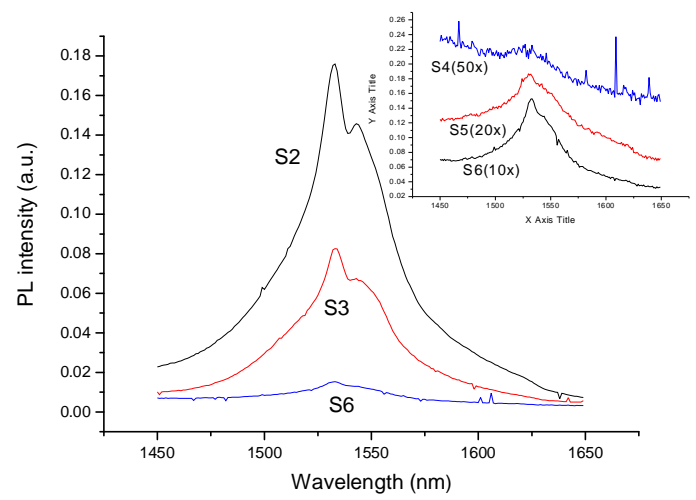

a)

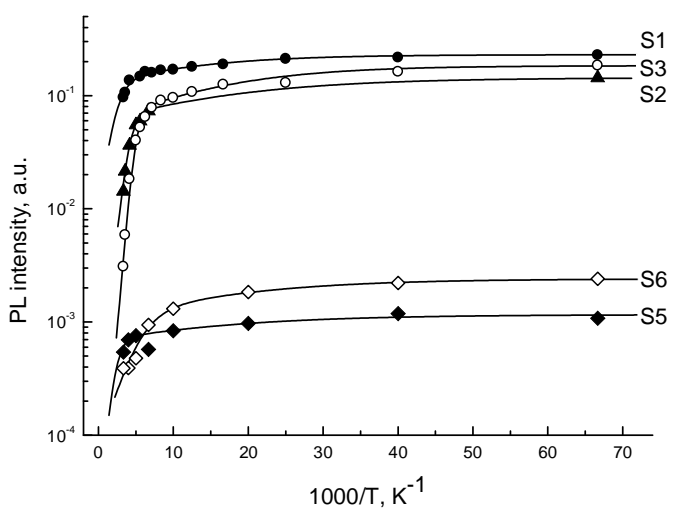

b)

Figure $3-$ a) PL spectra at $\sim 1.54 \mu \mathrm{m}$ measured at room temperature. b) $\mathrm{Er}^{3+}$ photoluminescence intensity as a function of temperature.

The temperature behavior was analyzed using the following fit function, which assumes a characteristic thermally activated excitation process, according to [18]:

$$
I(T)=I(0)\left[1+C_{1} \exp \left(-E_{1} / k T\right)+C_{2} \exp \left(-E_{2} / k T\right)\right]^{-1}
$$

where $E_{1}$ is the activation energy for the high temperature range and $E_{2}$ is the corresponding energy for the low temperature region. The coefficients $C_{1}$ and $C_{2}$ take into account the excitation processes of Er ions. Table II presents the corresponding fit parameters. 
Table II - Values of the curve-fitting parameters obtained for modeling temperature-dependent $\mathrm{Er}^{3+}-\mathrm{PL}$ behaviour using eq. (2).

\begin{tabular}{|c|c|c|c|c|c|}
\hline Sample & $\mathbf{C}_{\mathbf{1}}$ & $\mathbf{E}_{\mathbf{1}}(\mathbf{m e V})$ & $\mathbf{C}_{\mathbf{2}}$ & $\mathbf{E}_{\mathbf{2}}(\mathbf{m e V})$ & $\begin{array}{c}\text { PL at RT } \\
(\boldsymbol{\%})\end{array}$ \\
\hline S1 & 20 & 91 & 0.84 & 8 & 42 \\
\hline S2 & 800 & 125 & 1.42 & 6 & 10 \\
\hline S3 & 300 & 108 & 1.36 & 5 & 13 \\
\hline S6 & 350 & 97 & 0.85 & 4 & 8.7 \\
\hline
\end{tabular}

At room temperature the most intense $\mathrm{Er}^{3+} \mathrm{PL}$ was obtained in samples with a low fraction of small nanocrystals (S1, S2 and S3 samples), in agreement with the low temperature study. The percentage of Er PL at room temperature (RT) is $42 \%$ for S1 sample, around $15 \%$ for S2 and S3 samples and lower than $9 \%$ for the more crystalline ones as can be seen in Table II. We are able to conclude that not only the small Si nanocrystals/low crystalline volume fraction samples are better for the energy transfer to the Er ions, as also the temperature quenching is lower for the same samples.

Ours results also indicate that the $\mathrm{Er}^{3+} \mathrm{PL}$ quenching decreases with the increase of atomic-hydrogen content (see Table I and II samples S6, S2, S3 and S1). This decrease on the $\mathrm{Er}^{3+}$ PL quenching is probably due to a more complete hydrogen passivation of defects (at GB) that act as non-radiative recombination centers. It is well known that for the growth of good quality amorphous silicon thin films hydrogen is essential for improving their optical and electrical activity, since it will bind to silicon atoms saturating the silicon dangling bonds (DB). Therefore in nanocrystalline silicon thin films, hydrogen will be present in amorphous matrix and/or at the grain boundaries.

Photoluminescence decay traces were also recorded and Figure 4 shows the luminescence decay curve of the Er luminescence at $1536 \mathrm{~nm}$ for the $\mathrm{S} 1$ sample $(0.03$ at.\% Er) measured at $300 \mathrm{~K}$.

The Er decay time at $1536 \mathrm{~nm}$ (Fig. 4) is found to be $44 \mu$ s at $300 \mathrm{~K}$. This lifetime, being remarkable faster than the typical values for Er-doped $\mathrm{Si}$ based materials (about 2-20 $\mathrm{ms}$ in $\mathrm{SiO}_{2}$ or glasses; around 2-3 ms for porous $\mathrm{Si}$; about $1 \mathrm{~ms}$ crystalline $\mathrm{Si}$ ) is not yet understood. Typically a faster decay time is 
associated with the presence of strong non-radiative phenomena, probably related to the presence of dangling bonds (DB) or, for the case of samples with high Er content due to the energy transferred between neighbouring Er ions, since the Er-Er interactions are very strong.

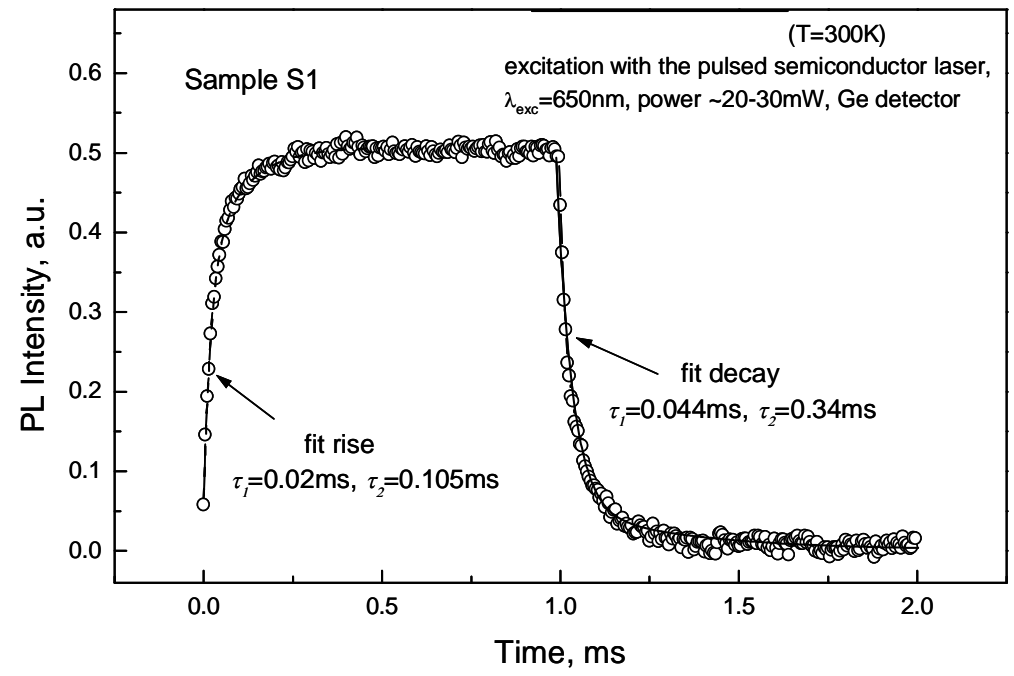

Figure 4 - Decay time of photoluminescence signal recorded at $1.54 \square \mathrm{m}$.

Furthermore, lifetimes lower than $44 \mu$ s are referred in the literature for as-grown or low-temperature annealed $\mathrm{Er}_{2} \mathrm{O}_{3}$ films [19]. In this way and since the hydrogen content of sample $\mathrm{S} 1$ is high enough for DB saturation and the erbium content is very low $(0.03 \mathrm{at} \%)$, probably the lifetime will increase towards reference values with sample thermal treatment.

\section{Conclusions}

Er-doped nanocrystalline silicon thin films have been grown by reactive magnetron r.f. sputtering under different conditions. Films with different crystal sizes, crystalline volume fraction and matrix chemical composition, in the as-grown form, have been obtained by changing the deposition parameters. 
The in-situ Erbium-doped nc-Si:H thin films were grown at low temperature and reveal Er PL at 1.54 $\mu \mathrm{m}$ in as-deposited form, without any further high-temperature annealing.

Erbium photoluminescence at $1.54 \mu \mathrm{m}$ without significant quenching up to temperatures $\sim 200-250 \mathrm{~K}$ was observed in RF-magnetron sputtered thin silicon films with different oxygen-to-hydrogen atomic concentration ratio, crystalline volume fraction and crystallite size. In these films, silicon nanocrystals are embedded in a-Si-H or oxygen-rich a-Si matrix whose nanostructure largely determines the photoluminescence efficiency and the chemical composition. The hydrogen content also plays an important role on the quenching effect.

With this work, some conclusions can be obtained, namely: (i) the $\mathrm{Er}^{3+} \mathrm{PL}$ is more intense for samples having small nanocrystals $(<5 \mathrm{~nm})$ and small crystalline volume fraction; (ii) We also confirm the results of others authors that the amount of Er optically active is small compared with its total amount; (iii) Our results also give evidence that the explanation of the small fraction of the erbium present in the samples being optically active is not only due to the fact of these ions are not close enough to the neighbouring Si atoms (sample S4 and S2 or S3 with the same Er, $\mathrm{H}$ and O), but due to the loss of excitation due to the proximity of $\mathrm{Si} \mathrm{NCs}$; (iv) The films produced showed excellent photoluminescence (PL) properties without the need of post-annealing treatment.

\section{Acknowledgements}

We acknowledge the financial support by FCT (POCTI/CTM/39395) and INTAS Project \#03-51-6486.

\section{References}

[1] - "Silicon-Based Optoelectronics", ed. by S. Coffa and L. Tsybeskov, MRS Bulletin 23, 16 (1998)

[2] -Y. Kanzawa, T. Kakevka, M. Fujii, S. Hayashi, K. Yamamoto, Solid State Commun. 102, 533 (1997) 
[3] - M. Fujii, M. Yoshida, Y. Kanzawa, S. Hayashi, K. Yamamoto, Appl. Phys. Lett., 71, 1198 (1997)

[4] - M. Fujii, M. Yoshida, S. Hayashi, K. Yamamoto, J. Appl. Phys. 84, 1 (1998)

[5] - G. Qin, G.G. Qin, S.H. Wanget., J. Appl. Phys., 85, 6738 (1999)

[6] - F. Priolo, G. Franzo, F. Iacona, D. Pacifici, V. Vinciguerra, Mat. Sc. Eng. B81, 9 (2001)

[7] - P. G. Kik, A. Polman, Mat. Sc. Eng. B81, 3 (2001)

[8] - D. Kuritsyn, A. Kozanecki, H. Przybylińska, and W. Jantsch, Appl. Phys. Lett. 83, 4160 (2003)

[9] - C. Garcia, P. Pellegrino, Y. Lebour, B. Garrido, F. Gourbilleau, R. Rizk, Journal of Luminescence 121, 204 (2006)

[10] - A.J. Kenyon, W.H. Loh, C.J. Oton, I. Ahmad, Journal of Luminescence 121, 193 (2006)

[11] - M. F. Cerqueira, M. Andritschky, L. Rebouta, J.A. Ferreira, M.F. da Silva, Vacuum 46, 1385 (1995)

[12] - C. Summonte, R. Rizzoli, A. Desalvo, F. Zignani, E. Centurioni, R. Pinghini, G. Bruno, M. Losurdo, P. Capezzuto, M. Gemmi, Philos. Magazine B, 80, 459 (2000)

[13] - I. Campbel and P.M. Fauchet, Solid State Commun. 58, 739 (1986)

[14] - Y. Kanemitsu, U. Uto et al, Physical Rev. B 48, 2827 (1993)

[15] - M. F. Cerqueira, M. Stepikhova, M. Losurdo, A. Kozanecki, T. Monteiro, Optical Materials, vol 28, pp. 836-841 (2006)

[16] -P.G. Kik, A. Polman, Materials Science and Engineering B81, 3-8 (2001)

[17] -P.G. Kik, A. Polman, J. Appl. Phys. 88, 1992 (2001)

[18] - H. Przybylinska, W. Jantsch, Yu. Suprun-Belevitch, M. Stepikhova, L. Palmetshofer, G. Hendorfer, A. Kozanecki, R. J. Wilson and B. J. Sealy, Phys. Rev. B 54, 2532 (1996)

[19] - M. Miritelo, R. Lo Savio, A.M. Piro, G. Franzo, F. Priolo, F. Iacona, C. Bongiorno, J. Appl. Phys. 100, 013502 (2006) 